UNITU-THEP-20/1997

\title{
Ward Identities for Yang-Mills Theory in Abelian Gauges: Abelian Dominance at High Energies
}

\author{
M. Quandt用, H. Reinhard棺 \\ Institut für Theoretische Physik, Universität Tübingen \\ D-72076 Tübingen, Germany.
}

\begin{abstract}
We consider Yang-Mills theory in a general class of Abelian gauges. Exploiting the residual Abelian symmetry on a quantum level, we derive a set of Ward identities in functional form, valid to all orders in perturbation theory. As a consequence, the coupling constant is only renormalised through the Abelian two-point function. This implies that asymptotic freedom in all Abelian gauges can be understood from an effective Abelian theory alone, which can be interpreted as Abelian dominance in the high energy regime.
\end{abstract}

\footnotetext{
*Supported by "Graduiertenkolleg: Hadronen und Kerne"

${ }^{\dagger}$ Supported in part by DFG under contract Re 856/1-3
} 


\section{Introduction}

In recent years, the possibility of realising confinement through a dual Meissner effect, as originally proposed by 't Hooft and Mandelstam [1], has triggered a vast variety of both analytical and numerical investigations. The magnetic monopoles necessary for the dual Meissner effect arise in the so-called Abelian gauges proposed by 't Hooft [2]. Recent lattice calculations [3] performed in these gauges have accumulated evidence in favour of Abelian dominance and the realisation of confinement through a dual Meissner effect. To be more precise, the lattice calculations performed in the maximal Abelian gauge show that about $95 \%$ of the full string tension are produced by Abelian gauge field configurations alone, of which about $90 \%$ are produced by magnetic monopoles [4. It is important to note that, in these lattice calculations, the sampling of the gauge field configurations is still done with the full Yang-Mills action, so that the non-Abelian field configurations are hidden in the weight with which the Abelian configurations contribute to, say, the partition function.

The Abelian, and in particular, monopole dominance is interpreted as supporting the dual superconductor picture of confinement. Furthermore, the Abelian dominance shows that confinement properties such as the string tension can be entirely obtained from an effective Abelian theory.

While the Abelian dominance in the string tension, i. e. in the low energy sector, has been intensively studied, little is known about possible Abelian dominance in the highenergy regime, in particular whether asymptotic freedom can be obtained from an effective Abelian theory. In the present paper we answer this question in the affirmative. We consider Yang-Mills theory in an arbitrary Abelian gauge. For the residual Abelian gauge symmetry we derive a set of Ward identities valid to all orders in perturbation theory. As a consequence of these Ward identities, the coupling constant is renormalised only through the Abelian two-point function, implying that in all Abelian gauges asymptotic freedom can be understood from an effective Abelian theory alone. This fact can be interpreted as Abelian dominance in the high energy regime. At one loop level and in the special case of the maximal Abelian gauge this fact was already observed in ref. [5] and subsequently confirmed in ref. [6].

\section{The Ward Identity in Abelian Gauges}

We consider pure $S U(N)$ Yang-Mills theory on an Euclidean four-manifold $\mathcal{M}$ with the action given by

$$
S_{\mathrm{YM}}[A]=\frac{1}{4} \int_{\mathcal{M}} d^{4} x F_{\mu \nu}^{a}(x) F_{a}^{\mu \nu}(x) .
$$

Here, $F_{\mu \nu}=F_{\mu \nu}^{a} T^{a}=\partial_{\mu} A_{\nu}-\partial_{\nu} A_{\mu}+g\left[A_{\mu}, A_{\nu}\right]$ denotes the field strength tensor of the gauge field $A_{\mu} \equiv A_{\mu}^{a} T^{a}, g$ is the coupling strength and the generators $T^{a}$ of the Lie algebra $\left[T^{a}, T^{b}\right]=f^{a b c} T^{c}$ are taken to be antihermitian and normalised according to $\operatorname{tr}\left(T^{a} T^{b}\right)=-\frac{1}{2} \delta^{a b}$

The basic idea of Abelian gauges is to remove as many non-Abelian degrees of freedom as possible by partially fixing the gauge, leaving a theory with a residual Abelian gauge symmetry. This is accomplished by the so-called Cartan decomposition, $G=H \times G / H$, where $G=S U(N)$ is the gauge symmetry group and $H \simeq U(1)^{N-1}$ denotes the maximal Abelian subgroup, spanned by a maximal set of commuting generators $\left\{T^{a_{0}}\right\}$. As a 
convention, we will use colour indices $a_{0}, b_{0}$ etc. to denote the generators of this Cartan subalgebra, while letters with a bar, $\bar{a}, \bar{b}$ etc. are reserved for the remaining generators.] The gauge field is then decomposed into its diagonal and off-diagonal parts,

$$
A_{\mu}(x)=A_{\mu}^{b_{0}} T^{b_{0}}+A_{\mu}^{\bar{b}} T^{\bar{b}} \equiv A_{\mu}^{(\mathrm{n})}+A_{\mu}^{(\mathrm{ch})} .
$$

The superscripts (ch) and (n) for "charged" and "neutral" refer to the transformation properties under the residual Abelian gauge group $\omega=e^{-g \theta^{b_{0}} T^{b_{0}}} \in H$ :

$$
A_{\mu}^{(\mathrm{n})} \rightarrow A_{\mu}^{(\mathrm{n})}+\frac{1}{g} \omega \cdot \partial_{\mu} \omega^{\dagger}=A_{\mu}^{(\mathrm{n})}+\partial_{\mu} \theta \quad ; \quad A_{\mu}^{(\mathrm{ch})} \rightarrow \omega \cdot A_{\mu}^{(\mathrm{ch})} \cdot \omega^{\dagger} .
$$

Under this residual symmetry, $A_{\mu}^{(\mathrm{ch})}$ transforms as a charged matter field in the adjoint representation, while the diagonal part $A_{\mu}^{(\mathrm{n})}$ behaves like a photon.

To fix the coset we need $N(N-1)$ conditions which leave the residual Abelian symmetry (3) unbroken. More specifically, we will consider charged gauge fixing conditions of the form

$$
\chi^{(\mathrm{ch})}[A] \equiv \chi^{\bar{a}}[A] T^{\bar{a}}=0 \quad ; \quad \chi^{(\mathrm{ch})}\left[A^{\omega}\right]=\omega \cdot \chi^{(\mathrm{ch})}[A] \cdot \omega^{\dagger},
$$

which are obviously invariant under the residual symmetry (3). This form includes all the commonly used Abelian gauges, in particular the "diagonalisation gauges" and the so-called maximal Abelian gauge.

The main motivation for the use of Abelian gauges is that, besides the appearance of magnetic monopoles, it should facilitate integrating out the charged gauge field components $A_{\mu}^{(\mathrm{ch})}$ leaving an effective Abelian theory. The quantisation of the latter still requires a gauge fixing for the neutral photon, which in the present paper will be done by the usual Lorentz condition

$$
\chi^{b_{0}} \equiv \partial_{\mu} A_{\mu}^{b_{0}}=0 .
$$

The complete gauge fixing can be regarded as a two-step process with the charged and neutral gauge conditions (4) and (5) being essentially independent of each other. This is justified by the standard Faddeev-Popov (or BRST) quantisation of the combined gauge (4,5). Relaxing the gauge conditions in the usual way by introducing gauge-fixing terms into the action, we obtain the generating functional in the form

$$
\begin{aligned}
Z\left[j^{(\mathrm{n})}, j^{(\mathrm{ch})}\right]= & \int \mathcal{D}\left(A^{(\mathrm{ch})}, A^{(\mathrm{n})}\right) \exp \left\{-\frac{1}{\hbar} S_{q}\left[A^{(\mathrm{n})}, A^{(\mathrm{ch})}\right]-\frac{1}{2 \hbar \alpha} \int d^{4} x\left(\partial_{\mu} A_{\mu}^{b_{0}}\right)^{2}+\right. \\
& \left.+\int d^{4} x j_{\mu}^{b_{0}} A_{\mu}^{b_{0}}+\int d^{4} x j_{\mu}^{\bar{b}} A_{\mu}^{\bar{b}}\right\} \\
S_{q}= & S_{\mathrm{YM}}+\frac{1}{2 \bar{\alpha}} \int d^{4} x \chi^{\bar{a}} \chi^{\bar{a}}-\hbar \operatorname{Tr} \ln \mathrm{M} .
\end{aligned}
$$

Here, $\alpha$ and $\bar{\alpha}$ are the gauge fixing parameters in the neutral and charged sector, respectively, and $\mathrm{M}$ denotes the Faddeev-Popov matrix. In the standard gauges, the non-Abelian nature of the Yang-Mills theory complicates the derivation of Ward identities from the functional integral. The problems are actually two-fold:

\footnotetext{
${ }^{1}$ For $G=S U(N)$ in the fundamental representation, we can always adjust the Cartan decomposition in such a way that the Abelian generators $T^{a_{0}}$ are diagonal and the remaining $T^{\bar{a}}$ have vanishing diagonal elements.
} 
Firstly, the variation of the gf. term under an infinitesimal gauge rotation involves the FP-matrix, leading to highly non-linear expressions in the resulting identities. Secondly, the FP-determinant calculated from

$$
\mathrm{M}^{a b}(x, y)=\left.\frac{\delta \chi^{a}\left[A^{\Omega}\right](x)}{\delta \varphi^{b}(y)}\right|_{\varphi=0} \equiv \mathrm{M}[A] \quad ; \quad \Omega=\exp \left(-g \varphi^{a} T^{a}\right)
$$

coincides with the gauge invariant Faddeev-Popov measure factor $\Delta$ only on the gf. manifold. This means that for arbitrary configurations $A$, the FP determinant $\operatorname{det} \mathrm{M}[A]$ will not be manifestly gauge invariant [7]. To see this more explicitly, we could follow the standard procedure and relax the gauge condition to $\chi^{a}[A]=c^{a}$. This will not alter the functional form of $\mathrm{M}$, but the gf. manifold and the measure factor $\Delta_{c}$ will clearly change. The gauge fixing delta function can now be removed by averaging over $c^{a}$ with a Gaussian weight, assuming that the $c$-dependence of $\Delta_{c}$ is eliminated by resolving the gf. constraint, $c^{a}=\chi^{a}[A]$. As a result, we find that $\operatorname{det} \mathrm{M}[A]$ is equivalent (for all configurations $A$ ) to $\Delta_{\chi[A]}$, and the implicit dependence on $A$ destroys the gauge invariance [7].

Due to these problems in the non-Abelian case, one cannot derive simple Ward identities directly based on the underlying gauge symmetry. Instead, one usually exploits the BRST invariance of the FP action leading to Slavnov-Taylor identities which are considerably more involved than their counterparts in QED. For the residual Cartan symmetry, however, we can return to simple Abelian relations even for the completely gauge fixed YM theory. This possibility is the key property of Abelian gauges. In fact, we will show that the quantum action $S_{q}$ of (6), including the (coset) gauge fixing term and the FP determinant, is invariant under the residual symmetry (3). From this point of view, YM theory in Abelian gauges is exactly equivalent to an Abelian system of photons and charged matter, although with a non-standard effective action.

Let us have a closer look at the behaviour of the quantum action (6) w.r.t. the Abelian transformation (3). The invariance of the coset gauge fixing term is a simple consequence of the transformation rule (4). We could even take this invariance as a definition for an Abelian gauge. As for the last term in $S_{q}$, i.e. the FP determinant, we have seen above that its invariance under the gauge rotation (3) is not obvious and, in fact, relies on the special property (車) of Abelian gauges. This can be seen as follows:

Let $\omega=e^{-g \theta^{b} T^{b_{0}}}$ be an arbitrary Abelian gauge transformation. In components, the gauge fixing constraints transform as

$$
\begin{aligned}
\chi^{a_{0}}\left[A^{\omega}\right] & =\mathrm{D}^{a_{0} b_{0}}[\omega] \cdot \chi^{b_{0}}[A]+\square \theta^{a_{0}}=\chi^{a_{0}}[A]+\square \theta^{a_{0}} \\
\chi^{\bar{a}}\left[A^{\omega}\right] & =\mathrm{D}^{\bar{a} \bar{b}}[\omega] \cdot \chi^{\bar{b}}[A],
\end{aligned}
$$

where

$$
\mathrm{D}^{a b}[\omega]=(-2) \operatorname{tr}\left(T^{a} \omega T^{b} \omega^{\dagger}\right)
$$

is the adjoint representation of the Abelian gauge rotation $\omega$. This quantity describes the transformation of a matter field in the adjoint representation, i.e. it is block-diagonal and unity in the neutral sector, whereas it constitutes a rotational matrix in the charged subspace. From the explicit calculation presented in appendix A we obtain the simple transformation law for the FP matrix (耳):

$$
\mathrm{M}\left[A^{\omega}\right]=\mathrm{D}[\omega] \cdot \mathrm{M}[A] \cdot \mathrm{D}^{T}[\omega]
$$


where $\mathrm{D}^{T}$ denotes the transpose of $\mathrm{D}$. In particular, this equation implies the invariance of $\operatorname{det} \mathrm{M}[A]$ under Abelian gauge rotations, since $\operatorname{det} \mathrm{D}=1$.

Let us also note that the Abelian and coset gauge fixing conditions become completely independent once we implement the latter exactly (see also ref. [5]). This can be achieved e.g. by replacing the coset gf. term in the quantum action $S_{q}$ by a Fourier representation of the FP (gauge fixing) delta function,

$$
\exp \left(-\frac{1}{2 \hbar \bar{\alpha}} \int d^{4} x \chi^{\bar{b}} \chi^{\bar{b}}\right) \rightarrow \int \mathcal{D} \phi^{(\mathrm{ch})} \exp \left(-\frac{i}{2 \hbar} \int d^{4} x \phi^{\bar{b}} \chi^{\bar{b}}\right)
$$

Here, the auxiliary multiplier field $\phi^{(\mathrm{ch})}$ has to transform like a charged matter field (cf. (14) under the residual symmetry in order to keep $S_{q}$ and the path integral measure invariant. We may then evaluate the FP determinant on the gf. manifold, where the factorisation into charged and neutral parts holds:

$$
\operatorname{det} \mathrm{M}_{\mathrm{FP}}=\operatorname{det}(-\square) \cdot \operatorname{det} \mathrm{M}_{\mathrm{FP}}^{\mathrm{ch}}
$$

Returning to eq. (16) for the generating functional, we have shown that the quantum action $S_{q}$ is always invariant under the Abelian transformation (3). This entails that the residual symmetry is only broken by the Abelian gauge fixing and the source terms. Exploiting this fact and the invariance of the path integral measure in (6), we can derive Ward identities in the usual way: We consider an infinitesimal change (3) of variables in the functional integral (which does not affect the value of $Z$ ) and set the variation of the Abelian gf. and source terms to zero.] Replacing fields by derivatives w.r.t. sources (when acting on the generating functional), we obtain eventually

$$
\left\{\frac{1}{\hbar \alpha} \partial_{\mu} \square \frac{\delta}{\delta j_{\mu}^{b_{0}}(x)}+\partial_{\mu} j_{\mu}^{b_{0}}(x)+g \cdot f^{\bar{a} b_{0} \bar{c}} j_{\mu}^{\bar{a}}(x) \frac{\delta}{\delta j_{\mu}^{\bar{c}}(x)}\right\} Z\left[j^{(\mathrm{n})}, j^{(\mathrm{ch})}\right]=0 .
$$

This result can be transformed into an identity for the effective action, i.e. the generating functional of 1PI Green's functions. Our conventions are as follows: We define the generating functional $W[j]$ of connected Green's functions by $Z[j]=\exp \left(-\hbar^{-1} W[j]\right)$. The usual Legendre transformation leads then to the classical field $A$ and the effective action $\Gamma[\mathrm{A}]$, respectively:

$$
\begin{aligned}
\mathrm{A}_{\mu}^{a}(x)[j] & =\left\langle A_{\mu}^{a}(x)\right\rangle_{j}=\frac{\delta}{\delta j_{\mu}^{a}(x)} \ln Z[j]=-\frac{1}{\hbar} \frac{\delta W[j]}{\delta j_{\mu}^{a}(x)} \\
\Gamma[\mathrm{A}] & =W[j[\mathrm{~A}]]+\hbar \int d^{4} x j_{\mu}^{a}[\mathrm{~A}] \mathrm{A}_{\mu}^{a} .
\end{aligned}
$$

With this convention, the Ward identity for $\Gamma$ becomes

$$
\frac{1}{\alpha} \square \partial_{\mu} \mathrm{A}_{\mu}^{b_{0}}(x)+\partial_{\mu} \frac{\delta \Gamma}{\delta \mathrm{A}_{\mu}^{b_{0}}(x)}+g \cdot f^{\bar{a} b_{0} \bar{c}} \frac{\delta \Gamma}{\delta \mathrm{A}_{\mu}^{\bar{a}}(x)} \mathrm{A}_{\mu}^{\bar{c}}(x)=0 .
$$

Let us note that we can easily extend this identity to include ghost fields $(\eta, \bar{\eta})$, if we prefer to represent the FP determinant in $S_{q}$ by a ghost integral in the usual way:

$$
\exp (\operatorname{Tr} \ln \mathrm{M}[A])=\int \mathcal{D}(\eta, \bar{\eta}) \exp \left(-\frac{1}{\hbar} \int \bar{\eta}^{a} \cdot \mathrm{M}^{a b}[A] \cdot \eta^{b}\right)
$$

\footnotetext{
${ }^{2}$ As usual, we discard the surface term in the variation of the Abelian source term.
} 
From the transformation property $(10)$ of the FP matrix, we infer that the ghosts have to be rotated as charged and neutral (scalar) matter fields w.r.t. the residual Abelian symmetry, i.e.

$$
\begin{array}{lll}
\eta^{a} \rightarrow \mathrm{D}^{a b}[\omega] \cdot \eta^{b} & ; & \eta=\eta^{a} T^{a} \rightarrow \omega \cdot \eta \cdot \omega^{\dagger} \\
\bar{\eta}^{a} \rightarrow \mathrm{D}^{a b}[\omega] \cdot \bar{\eta}^{b} & ; & \bar{\eta}=\bar{\eta}^{a} T^{a} \rightarrow \omega \cdot \bar{\eta} \cdot \omega^{\dagger} .
\end{array}
$$

In this way, the path integral measure and the quantum action $S_{q}$ (with the FP determinant replaced by the ghost term) will remain invariant under Abelian transformations. In addition, however, we will have to couple sources to the ghosts in order to include them in the effective action. The variation of these source terms under the gauge rotation (16) contributes to the Ward identity,

$$
\begin{aligned}
\frac{1}{\alpha} \square \partial_{\mu} \mathrm{A}_{\mu}^{b_{0}}(x)+\partial_{\mu} \frac{\delta \Gamma}{\delta \mathrm{A}_{\mu}^{b_{0}}(x)}+ & g \cdot f^{\bar{a} b_{0} \bar{c}}\left(\frac{\delta \Gamma}{\delta \mathrm{A}_{\mu}^{\bar{a}}(x)} \mathrm{A}_{\mu}^{\bar{c}}(x)+\right. \\
& \left.+\frac{\delta \Gamma}{\delta_{r} \eta^{\bar{a}}(x)} \eta^{\bar{c}}(x)-\bar{\eta}^{\bar{a}}(x) \frac{\delta \Gamma}{\delta_{l} \bar{\eta}^{\bar{c}}(x)}\right)=0 .
\end{aligned}
$$

The results (14, 17) are a direct consequence of the invariance of the quantum action (6) under Abelian gauge transformations and thus hold for all Abelian gauges.

\section{Renormalisation}

Let us now consider the consequence of the Ward identities (14), (17) for the renormalisation of our theory. The quantum corrections to the effective action, given by the difference between $\Gamma[\mathrm{A}](13)$ and the tree action (first and second term in the exponent of eq. (6)), can be expanded in powers of the fields [3

$$
\Delta \Gamma=\frac{1}{2} \mathrm{~A}^{(\mathrm{n})} \cdot \Pi^{(\mathrm{n})} \cdot \mathrm{A}^{(\mathrm{n})}+\frac{1}{2} \mathrm{~A}^{(\mathrm{ch})} \cdot \Pi^{(\mathrm{ch})} \cdot \mathrm{A}^{(\mathrm{ch})}+\frac{1}{2} g \mu^{\epsilon / 2} \delta G \cdot \mathrm{A}^{(\mathrm{ch})} \mathrm{A}^{(\mathrm{ch})} \mathrm{A}^{(\mathrm{n})}+\cdots
$$

We will assume that the vacuum polarisations $\Pi$ and the vertex correction $\delta G$ have been calculated to a given order in a loop expansion and divergent contributions are regularised in a gauge invariant way. The precise regularisation prescription is not important for the following, but in order to be specific in eq. (18), we use dimensional regularisation to $d=4-\epsilon$ Euclidean spacetime dimensions. Note that in this case, the cutoff $\epsilon \rightarrow 0$ is dimensionless, but an additional scale $\mu$ must be introduced to keep the regularised coupling constant dimensionless in $d \neq 4$.

The counterterms are constructed from the divergent part of the loop correction,

$$
\begin{aligned}
-\left.\Pi_{\mu \nu}^{(\mathrm{n}, \mathrm{ch})}(p, \mu, \epsilon)\right|_{\text {div,trans }} & =\delta Z^{(\mathrm{n}, \mathrm{ch})}(p, \mu, \epsilon) \cdot\left[D_{0,(\mathrm{n}, \mathrm{ch})}^{-1}\right]_{\mu \nu}^{\text {trans }}(p) \\
-\left.\Pi_{\mu \nu}^{(\mathrm{n}, \mathrm{ch})}(p, \mu, \epsilon)\right|_{\mathrm{div}, \text { long }} & =\delta Z_{(\alpha, \bar{\alpha})}(p, \mu, \epsilon) \cdot\left[D_{0,(\mathrm{n}, \mathrm{ch})}^{-1}\right]_{\mu \nu}^{\mathrm{long}}(p) \\
-\left.\delta G_{\mu \nu \rho}(p, \mu, \epsilon)\right|_{\mathrm{div}} & =\delta Z_{g}(p, \mu, \epsilon) \cdot\left[G_{0}\right]_{\mu \nu \rho}(p) .
\end{aligned}
$$

\footnotetext{
${ }^{3}$ For simplicity, we do not introduce ghost fields and use a shorthand notation where summation integration over all relevant indices is understood.
} 
Here, $D_{0}^{-1}$ and $G_{0}$ denote the (inverse) gluon propagator and triple gluon vertex, respectively, as obtained from expanding the initial action (6) in powers of the fields. If Furthermore, some renormalisation prescription to fix the finite parts in the factors $\delta Z$ is understood. Adding the counterterms to the initial action in (6) yields the same action in terms of the bare fields given by $\left(Z_{i}=1+\delta Z_{i}\right)$

$$
\begin{aligned}
& A_{B}^{(\mathrm{ch})}=Z_{\mathrm{ch}}^{1 / 2} \cdot A^{(\mathrm{ch})} \quad ; \quad A_{B}^{(\mathrm{n})}=Z_{\mathrm{n}}^{1 / 2} \cdot A^{(\mathrm{n})} \\
& \alpha_{B}=Z_{\alpha} Z_{\mathrm{n}} \cdot \alpha \quad ; \quad \bar{\alpha}_{B}=Z_{\bar{\alpha}} Z_{\mathrm{ch}} \cdot \bar{\alpha} . \\
& g_{B}=g \mu^{\epsilon / 2} \cdot Z_{g} Z_{\mathrm{ch}}^{-1} Z_{\mathrm{n}}^{-1 / 2}
\end{aligned}
$$

This bare action must now be used as starting point to calculate the loop corrections, and by construction, it will yield finite renormalised Green's functions when the bare quantities are re-expressed through the renormalised fields and $Z$-factors.

Let us denote by $\Gamma_{B}\left[A_{B}, g_{B}, \alpha_{B}\right]$ the effective action obtained in a loop expansion from the bare initial action. It is understood that $\Gamma_{B}$ is rendered finite by the same gauge invariant regularisation prescription that was already used for the extraction of the counterterms. Note that this also introduces a scale $\mu_{B}$ for the bare coupling $g_{B}$, which is however related to the scale $\mu$ in the counterterms by the renormalisation prescription. If we want e.g. the loop divergences in $\Gamma_{B}$ to be just cancelled (i.e. without finite parts) by the counterterms encoded in the $Z^{\prime} s$, we must have $\mu_{B}=\mu$.

Since the regularisation does not spoil gauge symmetry, we can derive a Ward identity on $\Gamma_{B}$ exactly as in (14). Replacing finally bare quantities by renormalised ones with the help of proper $Z$-factors, we obtain:

$$
\frac{1}{Z_{\alpha} \alpha} \cdot \square \partial_{\mu} \mathrm{A}_{\mu}^{b_{0}}(x)+\partial_{\mu} \frac{\delta \Gamma_{B}}{\delta \mathrm{A}_{\mu}^{b_{0}}(x)}+\frac{Z_{g}}{Z_{\mathrm{ch}}} \cdot g \mu^{\epsilon / 2} f^{\bar{a} b_{0} \bar{c}} \frac{\delta \Gamma_{B}}{\delta \mathrm{A}_{\mu}^{\bar{a}}(x)} \mathrm{A}_{\mu}^{\bar{b}}(x)=0 .
$$

Here, we have not distinguished between $\mu$ and $\mu_{B}$ since any deviation could be absorbed in the renormalisation prescription. It should be stressed once again that the bare effective action $\Gamma_{B}$ already contains the counterterms if re-expressed in terms of the renormalised quantities and $Z$-factors. Thus, differentiating $\Gamma_{B}$ w.r.t. the renormalised fields yields finite, renormalised Green's functions when the cutoff is removed $(\epsilon \rightarrow 0)$. As a consequence,

$$
\lim _{\epsilon \rightarrow 0} Z_{\alpha}(p, \mu, \epsilon)=\text { finite } \quad ; \quad \lim _{\epsilon \rightarrow 0} \frac{Z_{g}(p, \mu, \epsilon)}{Z_{\mathrm{ch}}(p, \mu, \epsilon)}=\text { finite }
$$

since all other quantities in (21) are finite as $\epsilon \rightarrow 0$. In the minimal subtraction scheme (and in practice also in all other commonly used regularisation prescriptions), this entails

$$
Z_{\alpha}(p, \mu, \epsilon)=1 \quad ; \quad \frac{Z_{g}(p, \mu, \epsilon)}{Z_{\mathrm{ch}}(p, \mu, \epsilon)}=1 .
$$

These considerations can be straightforwardly extended to the case where FP ghosts are introduced. The following additional relations between bare and renormalised quantities arise

$$
\begin{aligned}
& \eta_{B}^{(\mathrm{ch})}=\tilde{Z}_{\mathrm{ch}}^{1 / 2} \cdot \eta^{(\mathrm{ch})} \quad ; \quad \eta_{B}^{(\mathrm{n})}=\tilde{Z}_{\mathrm{n}}^{1 / 2} \cdot \eta^{(\mathrm{n})} \\
& \bar{\eta}_{B}^{(\mathrm{ch})}=\tilde{Z}_{\mathrm{ch}}^{1 / 2} \cdot \bar{\eta}^{(\mathrm{ch})} ; \quad \bar{\eta}_{B}^{(\mathrm{n})}=\tilde{Z}_{\mathrm{n}}^{1 / 2} \cdot \bar{\eta}^{(\mathrm{n})} \\
& g_{B}=g \mu^{\epsilon / 2} \cdot \tilde{Z}_{g} \tilde{Z}_{\mathrm{ch}}^{-1} Z_{\mathrm{n}}^{-1 / 2}
\end{aligned}
$$

\footnotetext{
${ }^{4}$ The precise definition of $D_{0}^{-1}$ and $G_{0}$ can be read off from eq. (27) below, with $\Gamma_{B}$ replaced by the initial quantum action (6).

${ }^{5}$ up to the loop order from which the counterterms were calculated.
} 
and from the universality of the renormalised coupling constant (as defined from the triple gluon or photon-ghost vertex), we infer

$$
\frac{\tilde{Z}_{g}(p, \mu, \epsilon)}{\tilde{Z}_{\mathrm{ch}}(p, \mu, \epsilon)}=\frac{Z_{g}(p, \mu, \epsilon)}{Z_{\mathrm{ch}}(p, \mu, \epsilon)}=1 .
$$

In fact, this relation also follows from the above considerations applied to the renormalised Ward identity including ghosts (cf. eq. (17)),

$$
\begin{aligned}
\frac{1}{Z_{\alpha} \alpha} \cdot \square \partial_{\mu} \mathrm{A}_{\mu}^{b_{0}}(x) & +\partial_{\mu} \frac{\delta \Gamma_{B}}{\delta \mathrm{A}_{\mu}^{b_{0}}(x)}+\frac{Z_{g}}{Z_{\mathrm{ch}}} \cdot g \mu^{\epsilon / 2} f^{\bar{a} b_{0} \bar{c}} \frac{\delta \Gamma_{B}}{\delta \mathrm{A}_{\mu}^{\bar{a}}(x)} \mathrm{A}_{\mu}^{\bar{b}}(x)+ \\
& +\frac{\tilde{Z}_{g}}{\tilde{Z}_{\mathrm{ch}}} \cdot g \mu^{\epsilon / 2} f^{\bar{a} b_{0} \bar{c}}\left(\frac{\delta \Gamma_{B}}{\delta_{r} \eta^{\bar{a}}(x)} \eta^{\bar{c}}(x)-\bar{\eta}^{\bar{a}}(x) \frac{\delta \Gamma_{B}}{\delta_{l} \bar{\eta}^{\bar{c}}(x)}\right)=0 .
\end{aligned}
$$

\section{Abelian Dominance in Asymptotic Freedom}

Let us briefly discuss the physical meaning of the Abelian Ward identities and the nonrenormalisation condition (23,25). As mentioned above, the derivatives of the bare regularised action w.r.t. the regularised fields are finite when the cutoff is removed. Transforming to momentum space, this entails that

$$
\begin{aligned}
\left.\frac{\delta^{2} \Gamma_{B}}{\delta \mathrm{A}_{\mu}^{a}(x) \delta \mathrm{A}_{\nu}^{b}(y)}\right|_{0} & =\int \frac{d^{d} p}{(2 \pi)^{d}} e^{-i p(x-y)}\left[D^{-1}\right]_{\mu \nu}^{a b}(p) \equiv \hbar\left\langle A_{\mu}^{a}(x) A_{\nu}^{b}(y)\right\rangle_{\text {ren }}^{-1} \\
\left.\frac{\delta^{3} \Gamma_{B}}{\delta \mathrm{A}_{\mu}^{a_{0}}(x) \delta \mathrm{A}_{\nu}^{\bar{b}}(y) \delta \mathrm{A}_{\rho}^{\bar{c}}(z)}\right|_{0} & =g \mu^{\epsilon / 2} \int \frac{d^{d}(p, q)}{(2 \pi)^{2 d}} e^{-i p(x-y)-i q(x-z)} \cdot i G_{\mu \nu \rho}^{a_{0} \bar{b} \bar{c}}(p, q)
\end{aligned}
$$

where the kernels $D^{-1}$ and $G$ depend on $p, \mu$ and the regulator $\epsilon$, but are finite when the cutoff is removed $(\epsilon \rightarrow 0)$. Note that $G$ is (up to a factor $-\hbar$ ) the momentum space representation for the 1PI triple gluon vertex with external lines removed, while $D$ is simply the gluon two-point function, as indicated above.

Ward identities for $D^{(\mathrm{n})}$ and $G$ can be easily derived from (21) by differentiating w.r.t. A and then setting all fields to zero. We find the colour structure

$$
\left[D^{(\mathrm{n})}\right]_{\mu \nu}^{\bar{a} \bar{b}}(p)=\delta^{\bar{a} \bar{b}} \cdot D_{\mu \nu}(p) \quad ; \quad G_{\mu \nu \rho}^{a_{0} \bar{b} \bar{c}}(p, q)=f^{a_{0} \bar{b} \bar{c}} \cdot G_{\mu \nu \rho}(p, q)
$$

to all orders in perturbation theory. Futhermore, the Ward identities constrain the longitudinal parts of the Green's functions. For the photon propagator, we have

$$
p_{\mu}\left[D^{(\mathrm{n})}\right]_{\mu \nu}^{-1}(p, \mu, \epsilon)-\frac{p^{2} p_{\nu}}{Z_{\alpha}(p, \mu, \epsilon) \alpha}=0 .
$$

Since all other quantities in this equation are finite as $\epsilon \rightarrow 0$, we find $Z_{\alpha} \equiv 1$ in the MS-scheme, which means that the neutral gauge fixing parameter is not renormalised in Abelian gauges. Note also that the longitudinal part of the solution to (29),

$$
D_{\mu \nu}^{(\mathrm{n})}(p, \mu, \epsilon \rightarrow 0)=\left(\delta_{\mu \nu}-\frac{p_{\mu} p_{\nu}}{p^{2}}\right) D^{(\mathrm{n})}\left(p^{2}, \mu\right)+\alpha \frac{p_{\mu} p_{\nu}}{p^{4}}
$$


is exhausted by the tree-level propagator, so that there are no quantum corrections to the longitudinal part of the two-point function. In particular, the photon vacuum polarisation must always be transversal. []

Turning to the vertex Ward identity, we obtain

$$
(p+q)_{\mu} G_{\mu \nu \rho}(p, q)+\frac{Z_{g}}{Z_{\mathrm{ch}}}\left(\left[D^{(\mathrm{ch})}\right]_{\nu \rho}^{-1}(q)-\left[D^{(\mathrm{ch})}\right]_{\rho \nu}^{-1}(p)\right)=0 .
$$

Once again, this implies $Z_{g}=Z_{\text {ch }}$ when the cutoff is removed (in the MS-scheme, this holds for all values of the regulator $\epsilon$ ). Notice that this can be interpreted as a form of Abelian dominance for perturbation theory and asymptotic freedom: The relation between bare and renormalised coupling in Abelian gauges is simply

$$
g_{B}=g \mu^{\epsilon / 2} \cdot Z_{\mathrm{n}}^{-1 / 2}(p, \mu, \epsilon)
$$

where $Z_{\mathrm{n}}$ is determined by the Abelian vacuum polarisation alone. This should not be taken to mean that charged fields can be neglected altogether, but we only need to consider diagrams with (two) neutral external photon lines in order to obtain the full $\beta$-function. Stated differently, we can extract asymptotic freedom from the correlator $\left\langle A^{(\mathrm{n})} A^{(\mathrm{n})}\right\rangle$ alone, where only the diagonal part of the field configuration enters, but these configurations are of course sampled with the full non-Abelian weight. This is similar to the phenomenon of Abelian dominance observed on the lattice for certain low-energy observables like the Wilson loop in the maximal Abelian gauge.

Let us finally mention that similar identities can be derived in the case that explicit ghost fields are introduced and the coupling constant is defined by the ghost-photon vertex. We may define scalar ghost propagators $D_{\operatorname{gh}}(p)$ and the ghost-photon vertex function $G_{\mu}(p, q)$ exactly as in (27) with the only difference that there is no Lorentz index for the scalar ghost fields. The corresponding Ward identity for the vertex is easily derived from (26), i.e.

$$
(p+q)_{\mu} G_{\mu}(p, q)+\frac{\tilde{Z}_{g}}{\tilde{Z}_{\mathrm{ch}}}\left[D_{\mathrm{gh}}^{-1}(p)-D_{\mathrm{gh}}^{-1}(q)\right]=0
$$

which again yields the cancellation $\tilde{Z}_{g}(p, \mu, \epsilon)=\tilde{Z}_{\mathrm{ch}}(p, \mu, \epsilon)$. In ref. [5], an explicit calculation in the maximal Abelian gauge confirmed the validity of the Ward identity (33) and the cancellation $\tilde{Z}_{g}=\tilde{Z}_{\text {ch }}$ to one loop order:

$$
\tilde{Z}_{\mathrm{ch}}(p, \mu, \epsilon)=\tilde{Z}_{g}(p, \mu, \epsilon)=1+\hbar g^{2} \frac{3-\alpha}{8 \pi^{2} \epsilon}+\mathcal{O}\left(\hbar^{2}\right)
$$

Note that the counterterms (and thus the $Z$-factors) are independent of $p$ and $\mu$ in the MS scheme. Furthermore, the effective action of [5] yields a transversal photon vacuum polarisation with

$$
Z_{\mathrm{n}}(p, \mu, \epsilon)=1+\hbar g^{2} \frac{11 \kappa}{24 \pi^{2} \epsilon}+\mathcal{O}\left(\hbar^{2}\right) \quad ; \quad \kappa=C_{2}(S U(2))=2
$$

From (32) this gives indeed the correct one-loop $\beta$-function for $G=S U(2)$ from Abelian correlators alone.

\footnotetext{
${ }^{6}$ This is of course a consequence of the Lorentz gauge in the Abelian sector, but it holds independently of the coset gf. condition, i.e. also for non-linear gauges like the maximal Abelian gauge.
} 


\section{Conclusions}

Abelian gauges generally help to reduce Yang-Mills theory to a (non-standard) Abelian model of photons and charged matter. In the present work we have studied this reduction on a quantum level. While the Cartan decomposition initially complicates the theory (there are more renormalisation constants), this is compensated by gaining simple symmetry relations in the Abelian sector. As a consequence, Abelian gauges are very useful whenever the physics under consideration can essentially be described by the effective Abelian theory.

On the lattice, such an Abelian dominance has been observed approximately in the low energy regime, but only for special gauges such as the maximal Abelian gauge. In the present work we have investigated the use of Abelian gauges in the asymptotic freedom regime. We find that Ward identities associated with the residual Abelian symmetry put stringent restrictions on some of the Green's functions, leading to powerful relations between the renormalisation constants. More precisely, we have proved that in any Abelian gauge, the correct perturbative $\beta$-function of Yang-Mills theory can be obtained from the Abelian gluon propagator alone. The effects of the non-Abelian gauge fields are entirely absorbed in the arising effective Abelian gluon propagator. This result can be interpreted as Abelian dominance in the high-energy regime. We are thus led to the conclusion that Abelian gauges can be convenient not only in the confinement region, but also in the asymptotic freedom regime.

\section{Acknowledgment}

We would like to thank R. Alkofer and M. Engelhardt for carefully reading the manuscript and helpful discussions.

\section{A Transformation Property of the FP Matrix}

We start from the definition (7) of the FP matrix with a gauge rotated argument,

$$
\mathrm{M}^{a b}\left[A^{\omega}\right]=\left.\frac{\delta \chi^{a}\left[\left(A^{\omega}\right)^{\Omega}\right]}{\delta \varphi^{b}}\right|_{\varphi=0}=\left.\frac{\delta \chi^{a}\left[\left(A^{\tilde{\Omega}}\right)^{\omega}\right]}{\delta \varphi^{b}}\right|_{\varphi=0}
$$

where $\omega=e^{-g \theta^{b} 0 T^{b_{0}}}$ denotes an arbitrary Abelian gauge transformation and $\Omega=e^{-g \varphi^{b} T^{b}}$. In the second step, we have introduced

$$
\tilde{\Omega}=\omega^{\dagger} \cdot \Omega \cdot \omega=e^{-g \tilde{\varphi}^{c} T^{c}} .
$$

This allows us to apply the special transformation properties (8) of Abelian gauges, $\chi^{a}\left[A^{\omega}\right]=\mathrm{D}^{a b}[\omega] \cdot \chi^{b}[A]+$ const $[\omega]$, with the rotational matrix $\mathrm{D}$ defined in (9). Upon applying the functional chain rule, we find

$$
\mathrm{M}^{a b}\left[A^{\omega}\right]=\left.\left.\mathrm{D}^{a c}[\omega] \cdot \frac{\delta \chi^{c}\left[A^{\tilde{\Omega}}\right]}{\delta \tilde{\varphi}^{d}}\right|_{\varphi=0} \cdot \frac{\delta \tilde{\varphi}^{d}}{\delta \varphi^{b}}\right|_{\varphi=0} .
$$

From the definition (37), we observe that $\Omega=\mathbf{1} \Longleftrightarrow \tilde{\Omega}=\mathbf{1}$, or $\varphi=0 \Longleftrightarrow \tilde{\varphi}=0$. The second factor in (38) is then simply the FP matrix evaluated at the non-rotated argument 
$A_{\mu}$. To complete the proof of the transformation property (10) quoted in the main text, we only have to show that

$$
\left.\frac{\delta \tilde{\varphi}^{a}}{\delta \varphi^{b}}\right|_{\varphi=0}=(-2) \operatorname{tr}\left(T^{b} \omega T^{a} \omega^{\dagger}\right)=\mathrm{D}^{b a}[\omega]
$$

which follows, after a simple calculation, from the definition (37).

\section{References}

[1] G. 't Hooft, in High Energy Physics EPS Int. Conference, Palermo 1975, ed. A. Zichichi, Physica Scripta 25 (1982) 133.

S. Mandelstam, Phys. Rep. C23, (1976) 245.

[2] G. 't Hooft, Nucl. Phys. B190[FS3] (1981) 455.

[3] A.S. Kronfeld, G. Schierholz, U.-J. Wiese, Nucl. Phys. B293 (1987) 461. for review talks see:

T. Suzuki, Nucl. Phys. Proc. Suppl. B30 (1993) 176.

A. Di Giacomo, preprint, hep-lat/9509036.

M. Polikarpov, Nucl. Phys. Proc.Suppl. B53 (1997) 134.

[4] G.S. Bali, V. Bornyakov, M. Mueller-Preussker, K. Schilling, Phys. Rev. D54 (1996) 2863.

[5] M. Quandt, H. Reinhardt, "Field Strength Formulation of $S U(2)$ Yang-Mills Theory in the Maximal Abelian Gauge: Perturbation Theory.", hep-th/9707185.

[6] K. Kondo, preprint, hep-th/9709109.

[7] R.J. Rivers, "Path Integral Methods in Quantum Field Theory", Cambridge University Press, 1987. 